\title{
EDUCACIÓN Y CONOCIMIENTO: ¿EJE DE LA TRANS- FORMACIÓN PRODUCTIVA CON EQUIDAD? EL CASO DE CHILE
}

\section{(Education and Knowledge: The core of productive transformation with equity?. The case of Chile)}

\section{Article record \\ $\underline{\text { About authors }}$ \\ $\underline{\text { HTML format }}$}

\section{por} \\ Ilich Silva-Peña (isilva@accioneducativa.cl) \\ Alexis Chelme-Bustos (aachelme@puc.cl) \\ Isabel Salgado-Labra (isalgado@accioneducativa.cl)
}

Ficha del artículo

$\underline{\text { Sobre los autores }}$

Formato HTML

\begin{abstract}
The objective of this article is to give a review of the results of educational politics in Chile, of which the core concept has been "productive transformation with equity".

It has established a view on the aspects of productivity and distribution of income in the different socioeconomic stratums, and it shows the levels of education achieved in comparison to the incomes earned.

Among the principle conclusions that were found, it is certain that even though productivity has increased, equity has not, which shows problems with educational development in Chile
\end{abstract}

\section{Keywords}

Education, Chile, Educational Policy, Educational Reform, Economy of the education, Equity of the Education

\section{Resumen}

El presente artículo tiene por objetivo realizar una revisión de los resultados de políticas educativas en Chile, que han tenido como eje central el concepto de "transformación productiva con equidad".

Se establece una mirada desde los aspectos de la productividad y la distribución de ingresos en los distintos estratos socioeconómicos, realizando una comparación de los niveles educativos alcanzados de acuerdo al nivel de ingreso.

Dentro de las principales conclusiones y resultados se encuentra que, si bien es cierto, la productividad ha tenido un aumento, esto no ha sido así en los aspectos de equidad, lo cual podría señalar algunos inequívocos del desarrollo educativo en Chile.

\section{Descriptores}

Educación, Chile, Política Educativa, Reforma Educativa, Economía de la Educación, Equidad en Educación

\section{Introducción}

Como título de este trabajo se ha puesto en interrogante el texto de la Comisión Económica para América Latina (CEPAL,1990), que publicara a principio de los noventa como "Educación y conocimiento: Eje de la transformación productiva con equidad", de tal manera de preguntarse cuál ha sido el desarrollo de dicha propuesta.

En el libro antes señalado se estimaba como vía de desarrollo para los países latinoamerica- 
Silva-Peña, I., Chelme-Bustos, A. y Salgado-Labra, I. (2003). Educación y conocimiento:¿Eje de la transformación productiva con equidad? El caso de Chile. RELIEVE:, v. 9, n. 1, p. 73-81.

http://www.uv.es/RELIEVE/v9n1/RELIEVEv9n1 3.htm

nos la competitividad internacional basada fundamentalmente en el fortalecimiento de la base empresarial, la infraestructura tecnológica, la apertura internacional y muy especialmente la formación de recursos humanos y el conjunto de incentivos y mecanismos que favorecen el acceso a la generación de nuevos conocimientos. (CEPAL, 1992). Este trabajo será continuador de las propuestas generadas en el marco de la denominada "Transformación productiva con equidad", conjunto de recomendaciones diseñadas por la CEPAL en torno a generar un marco de crecimiento económico en América Latina y el Caribe que acompañado de políticas sociales pudiese crear un marco de desarrollo entendido como un "crecimiento con equidad", comprendiendo a éste como un crecimiento en el cual participara toda la población y fundamentalmente tendiendo a la disminución de la pobreza (CEPAL, 1990).

En Chile, a principio de los 90, los gobiernos de la Concertación de Partidos por la Democracia trazaron el camino económico a seguir durante la última década del siglo XX y el comienzo del siglo XXI, diseño realizado en base a las recomendaciones antes mencionadas.

Como desafíos se propone por un lado la mayor incorporación en el escenario de competitividad internacional, a través de productos con mayor nivel de elaboración, mejorando la calidad y cantidad de sus exportaciones y modernizando su aparato productivo. Por otra parte, se plantea avanzar en el mejoramiento de la calidad de vida de todos los chilenos y la superación de la pobreza. "Con este fin, el estado promueve la inversión, para mejorar el recurso humano y crear las condiciones físicas que permitan que el trabajo sea más productivo $\mathrm{y}$, por lo tanto, genera mayor ingreso" (MIDEPLAN, 1992), es decir el recurso humano se pone al centro del desarrollo productivo, enfocado como el principal gestor de la transformación productiva con equidad.

En el ánimo de generar cambios a nivel de los recursos humanos, se establecen diseños de políticas a nivel educativo, dentro de las cuales se conforma un comité que elabore propuestas para la modernización la educación, entendida ésta como el pilar para hacer un país competitivo, el cual a través del crecimiento económico puede resolver diversos problemas, entre ellos el más importante: la pobreza (COMITÉ, 1995).

En el caso del comité técnico asesor (1995) nos señala que "las personas pobres sólo tienen a disposición su propia capacidad de trabajo. De allí que la educación sea su mayor aspiración y el medio más eficaz para cambiar sus condiciones de vida. Ellos saben que a mayor y mejor educación aumentan las posibilidades de percibir remuneraciones más altas" (COMITÉ, 1995:64)

Por lo tanto sería a través de un mayor nivel educativo que conseguirían un aumento en sus remuneraciones, cual produciría por una parte un país más competitivo y por otro lado un país más equitativo.

Esta visión establece entonces que la transformación productiva con equidad sólo se puede dar a través de la educación, de esta manera a principios de los 90 se comienza con un periodo de reformas educativas que comienzan con la educación básica, extendiéndose a la educación media y superior, tomando en consideración de que la cobertura educativa si bien no estaba plenamente satisfecha había un logro bastante notable en cuanto a cobertura a nivel básico que llegaba a un 96,6 \% y de educación media que era de $80,5 \%$, no así en cuanto a la educación parvularia, cuya cobertura alcanzaba el 20,9 para niños y niñas de 0 a 5 años, aunque en el tramo de 0 a 2 años sólo era de un 2,5\% (MIDEPLAN, 1999:19)

La idea de este artículo es comenzar la revisión de algunos resultados a los cuales se ha llegado en la aplicación de estas políticas, especialmente se pretende describir el estado actual de la equidad en cuanto a los ingresos monetarios y su relación con la productividad, y a la vez encaminarse un análisis hacia el rol de la educación en este periodo. 
Silva-Peña, I., Chelme-Bustos, A. y Salgado-Labra, I. (2003). Educación y conocimiento:¿Eje de la transformación productiva con equidad? El caso de Chile. RELIEVE:, v. 9, n. 1, p. 73-81.

http://www.uv.es/RELIEVE/v9n1/RELIEVEv9n1 3.htm

\section{Productividad - Masa de Remuneracio- nes.}

Según la información entregada por el MIDEPLAN (2001a) El crecimiento de las remuneraciones reales entre 1989 y 2000 fue de un $3,6 \%$ anual, en tanto que en ese mismo informe se señala que el crecimiento de la productividad (calculado bajo la base de PIB/ ocupados ) fue de un $4,2 \%$ durante el mismo periodo, presentando una acumulación en el crecimiento de las remuneraciones de 147,7 tomando $1989=100$, es decir el crecimiento de las remuneraciones entre 1989 y 2000 fue de un $47,7 \%$ acumulado, por otra parte la índice de productividad creció de $100=1989$ a un 161,5 es decir un crecimiento de $61,5 \%$.

Debemos tomar en cuenta que en el total de las remuneraciones están contabilizadas desde el salario mínimo hasta los sueldos de gerentes o ejecutivos de empresas transnacionales y en el índice de productividad se señala tanto las grandes empresas como las pequeñas y microempresas en las que el trabajador es el dueño de la empresa.

Durante los años 1989 y 2000 la diferencia entre el aumento de la productividad, es decir el PIB/ ocupados y el aumento del índice real de remuneraciones fue de $13.8 \%$ y si establecemos una relación proporcional podríamos decir que por cada 1 punto de variación en el índice de remuneraciones hubo un 1,29 \% de variación en la productividad, con esto se señala que si la relación remuneraciones/productividad la trasladamos al año 2000 podríamos establecer que el conjunto de trabajadores y trabajadoras produce casi un $30 \%$ más.

\section{Distribución personal del ingreso}

Si tomamos en consideración el reparto de ingresos a través de la distribución por quintil del ingreso autónomo nacional (MIDEPLAN, 2000), podemos señalar que entre los años 1996 y 2000 no existe ninguna diferencia, puesto que en 1996 el quintil de menores ingresos tuvo una participación del 4,2\% del ingreso autónomo nacional , mientras que el año 2000 ese porcentaje se mantuvo estable en los $4,2 \%$; en cuanto al quintil de mayores ingresos, tuvieron una participación de $56,6 \%$ del total en 1996 , mientras que en el año 2000 esta participación sube a un 56,9\%, subiendo así el quintil de mayores ingresos en un $0,3 \%$ de la participación del ingreso autónomo nacional ${ }^{[1]}$.

Si comparamos esta situación entre los años 1996 y 2000 vemos que no existe una gran variación en el quintil de menores ingresos, pero si establecemos esa relación con el año 1992 podemos decir que existe un empobrecimiento del primer quintil puesto en ese año este sector de la población participaba en un 4,92\% del ingreso según un informe de la CEPAL (citado por Agasino, 1996), mientras que el último quintil participaba de un 55,45\% del ingreso autónomo nacional, podemos decir entonces que estos últimos años sólo se ha tratado de paralizar esta creciente inequidad entre los quintiles primero y último, pero si tomamos en consideración que entre 1992 y 2000 el gobierno y las políticas económicas han sido dirigidas por la misma coalición política (concertación de partidos por la democracia), podemos expresar que en el desarrollo socio-económico se ha dado una creciente inequidad entre los quintiles extremos.

Dentro de esta revisión económica podríamos señalar que no importa que en una década pueda existir un pequeño detrimento del sector de menores ingresos de la población, siempre y cuando existieran luces de que esto variará en el tiempo y que finalmente se podrá obtener una situación en la que exista una mayor equidad en la distribución de los ingresos, he ahí la importancia que podría tener la educación como un vehículo que permita acercarse a mejores niveles de justicia económica y social.

\section{Educación como solución de la des- igualdad}

El tema planteado por la CEPAL en torno a ser la educación una generadora de crecimiento en la economía es un elemento hasta ahora poco cuestionado, de hecho esta misma institución plantea que la inversión realizada en edu- 
Silva-Peña, I., Chelme-Bustos, A. y Salgado-Labra, I. (2003). Educación y conocimiento:¿Eje de la transformación productiva con equidad? El caso de Chile. RELIEVE:, v. 9, n. 1, p. 73-81.

http://www.uv.es/RELIEVE/v9n1/RELIEVEv9n1 3.htm

cación puede verse reflejada en el " aumento de conocimientos y destrezas que aumentan la productividad del trabajo. El crecimiento del producto nacional (beneficio para el país) y del ingreso (beneficio para el individuo) La disminución de tiempo que se requiere para el acceso al mercado laboral, lo que beneficia tanto al individuo como a la sociedad" (Cohen,1997)

Si tomamos en consideración los aspectos anteriores podríamos señalar que la inversión en educación realizada (del 2.6 gasto social educación/PIB entre 90-91 a 3.3 96-97) según la CEPAL (2000), ha sido rentabilizada puesto que efectivamente se ha aumentado la productividad en los últimos años y también han crecido las remuneraciones, pero sin embargo como veíamos anteriormente no se ha acortado la brecha entre quienes ganan más y quienes tienen menores ingresos, muy por el contrario, esa brecha ha aumentado levemente.

\section{La equidad en educación}

El Ministerio de Educación de Chile MINEDUC (1997) establece que uno de los elementos que puede generar un estado de mayor equidad es el desarrollo educativo, es por esto que las oportunidades de acceso a la educación se han puesto en el centro de la discusión como un factor clave, lo que ha generado diversas políticas públicas en torno a ampliar cobertura y mejorar la calidad de la educación.

Otro de los elementos presentes ha sido el logro de la equidad en educación abordada desde un punto de vista intrasistema en el cual alude al grado de homogeneidad de la calidad en la oferta educativa que atiende distintos estratos socioeconómicos, siendo este el enfoque que ha primado en las reformas educativas latinoamericanas (CEPAL, 1997)

Podemos decir entonces que la mirada de la equidad en educación ha sido entendida en dos aspectos, uno: aumentar la cobertura educacional y otro el desarrollo de una educación de calidad para todos.
La mirada de este trabajo está puesta principalmente en la relación existente de 2 niveles educativos, la Educación Preescolar y la Educación Superior, los cuales no gozan de las cifras de acceso educativo que tienen los otros niveles, puesto que el acceso a la Educación Básica es obligatorio, por tanto la cobertura llega a cifras cercanas al $100 \%$ y en el caso de la educación Media si bien es cierto no se cubre totalmente el $100 \%$, pero existe un $82,3 \%$ de cobertura en el $20 \%$ más pobre, mientras que el quintil de mayor ingreso hay un 98,5\% de acceso a este nivel educativo, en estos casos a pesar de existir diferencias no son tan notorias como en los otros niveles, pero si es cierto que estas cifras influirán en el acceso a la educación superior, tal como lo veremos más adelante.

\section{Educación Preescolar:}

El acceso a la educación Preescolar ha sido considerada como un elemento que puede desequilibrar la equidad en los ingresos monetarios, estudios revelan que programas efectivos, dirigidos a esta etapa inicial de la vida de los niños, tienen un impacto significativo en mejorar el rendimiento de éstos durante la enseñanza básica y media, además de una mayor productividad y mejores ingresos en su vida laboral (Young citado por UNICEF, 2000).

Si partimos de la base que el acceso a la educación puede mejorar la distribución de ingresos en los hogares chilenos debiéramos fijar un poco nuestra mirada a la evolución de la cobertura de este nivel educativo.

En el año 1990 la cobertura de educación parvularia en el primer quintil de ingreso autónomo nacional fue de un $16,9 \%$, aumentando a $25,5 \%$ en el año 2000 , es decir un aumento de $8,6 \%$ en 10 años. En el caso del quintil de mayores ingresos el aumento de cobertura en el nivel de educación parvularia fue de $32,4 \%$ a $50,2 \%$ esto señala un aumento de $17,8 \%$, poco más del doble que en el primer quintil.

Podemos decir entonces que en la educación parvularia se ha aumentado la brecha de acce- 
Silva-Peña, I., Chelme-Bustos, A. y Salgado-Labra, I. (2003). Educación y conocimiento:¿Eje de la transformación productiva con equidad? El caso de Chile. RELIEVE:, v. 9, n. 1, p. 73-81.

http://www.uv.es/RELIEVE/v9n1/RELIEVEv9n1 3.htm

so, con lo cual están en desventaja las familias de menores ingresos en relación a las familias que tienen mayores ingresos, esto plantea la posibilidad de acceder a incrementar el ingreso familiar por parte de aquellas madres que pertenecen a los niveles económicos más altos, ya que cuentan con un espacio donde se mantiene a su hijo o hija, lo que les permite ampliar su desarrollo laboral, pero lo que más debe concitar la atención es que al existir una creciente brecha entre el acceso del $20 \%$ más pobre en comparación con el $20 \%$ más rico de la población, nos vemos enfrentados a una situación futura en la que esta diferencia en el ingreso aumentará de manera considerable al no existir medidas correctoras en los niveles educativos próximos.

\section{Educación Superior:}

En el caso de la Educación Superior en el primer quintil, el acceso a la educación Superior creció de un 4,4\% en 1990 a un $9,4 \%$ durante el 2000, es decir 5 puntos porcentuales. En cambio en el quintil de mayores ingresos presentó un crecimiento de $40,2 \%$ a $65,6 \%$ durante la misma década, esto señala un aumento de $25,3 \%$, lo que representa más de 5 veces el incremento del quintil de menores ingresos (MIDEPLAN, 2001b)

En este punto queda claro que la brecha de acceso a la educación superior se ha incrementado en el último tiempo, aunque podemos señalar que dentro de esta diferencia no se realiza una diferenciación entre quienes ingresan a un CFT (Centro de formación técnica), IP (Instituto Profesional) o Universidades (ya sean privadas o públicas), puesto que al revisar la diferencia entre quintiles de acuerdo a esta segmentación podemos ver que del $100 \%$ de cobertura de las universidades pertenecientes al consejo de rectores ( o llamadas también tradicionales) sólo el 5,3 $\%$ pertenece al quintil de menores ingresos, mientras que el 39,4 \% forma parte del quinto quintil. En tanto en la Educación que se realiza en los Centros de Formación Técnica la presencia del primer quintil es de $8,4 \%$ en comparación con el $100 \%$ que asiste ese tipo de educación, en tanto el $18,4 \%$ corresponde al quintil de mayores ingresos.

Con lo anterior podríamos señalar que hay un mayor acceso a la Universidad de las familias de mayores ingresos, lo que significa que en un futuro pueden acceder a mejores empleos ya que en estas instituciones entregan títulos profesionales que requieren una preparación de 4 años o más, en cambio en los CFTs se entrega la preparación de los títulos técnicos que requieren 2 o menos años de preparación.

Si tomamos en consideración que según la CEPAL en el año 1998 en Chile los años de escolaridad necesarios para tener un $90 \%$ o más posibilidades de no caer en la línea de la pobreza era entre 12 y 14 años (CEPAL, 2000) , se estima que el gran porcentaje de aquellos jóvenes que pertenecen a familias del quintil de menores ingresos estarían bordeando la línea de la pobreza, a pesar de que hoy día hay más acceso a la educación superior por parte de las personas pertenecientes a este quintil.

Lo anterior no toma en consideración la devaluación de la educación, esto significa que a igual puesto de trabajo hoy se aumenten las exigencias de nivel educativo, por tanto es posible que haya una variación en cuanto a las diferencias que se aprecian lo cual aumentaría aún más la inequidad, aunque no es posible precisar cuanto en este trabajo.

Debido que el ingreso a la Educación Superior no es inmediato, puesto que se necesita de la aprobación de la Educación Media, podríamos decir que el aumento en el acceso a la Educación Media en el primer quintil que fue de 73,3 en 1990 a 82,3 el 2000, puede haber influido e el avance del acceso la educación superior que ha tenido dicho segmento. Si consideramos que este aumento no depende solamente del aumento de infraestructura, son que más bien de la continuidad de estudios en la educación, podemos decir que ha existido un avance e la equidad intrasistema, puesto que hoy día aumentan las posibilidades de acceso gracias a la entrega de una educación de mejor calidad. 
Silva-Peña, I., Chelme-Bustos, A. y Salgado-Labra, I. (2003). Educación y conocimiento:¿Eje de la transformación productiva con equidad? El caso de Chile. RELIEVE:, v. 9, n. 1, p. 73-81.

http://www.uv.es/RELIEVE/v9n1/RELIEVEv9n1 3.htm

Pero si bien es cierto significa un aumento en el ingreso del primer quintil, no sería correcto decir que hemos superado la inequidad existente, muy por el contrario esta ha aumentado a pesar de los esfuerzos institucionales.

Al analizar el conjunto de estos datos podríamos señalar, tal como los franceses Baudelot y Establet (1998) que el nivel educativo aumenta, pero no para todos, puesto que no basta con analizar los promedios ya que el motor de la dinámica está el alojado en quienes perciben mayores ingresos y esto disimula la inequidad.

\section{Comentarios finales}

Si tomamos en consideración las variaciones de la productividad en relación con los salarios podemos ver que existe una pérdida por parte de trabajadores y trabajadoras quienes de alguna manera están produciendo más con un mismo ingreso, entendido este con su actualización.

Por otra parte tenemos un claro aumento en la diferencia que existe en la participación del ingreso autónomo nacional entre el quintil de menores ingresos y el de mayores ingresos, aunque en los últimos 4 años esto parece tener cierta estabilidad, el resultado de la década es desfavorable para el quintil de menores ingresos.

De alguna manera podemos decir que si ha aumentado la productividad, pero también ha aumentado la inequidad. Quizás lo más preocupante no sólo es la desigualdad económica existente, sino que lo que puede seguir a futuro, si tomamos en cuenta que la inequidad en los ingresos va aumentando no hay nada que indique que esta situación se podría revertir.

Tal como lo expresa Martinic (2000) en general se ha asumido más un discurso en pro de la calidad que de la equidad, discurso que predomina a favor de la mejorar la competitividad y la excelencia, pero al parecer esto no sería lo más apropiado para los países de América Latina puesto que lo que se necesita es poner énfasis en tanto en la equidad como en la calidad si queremos resolver los problemas de desigualdades sociales.
Si siguiéramos la perspectiva hasta ahora desarrollada podríamos señalar que la calidad tiene un efecto en la productividad puesto que en el marco del desarrollo de la experiencia chilena se ha aumentado la productividad y las cifras macroeconómicas han tenido un notable avance. Esto no significa que haya disminuido la inequidad, por tanto se vuelve a pensar en la tesis de que para lograr un crecimiento equitativo no basta con crecer sino que se deben aplicar políticas públicas en favor de una mejor distribución.

Pero si tales políticas son las que hasta el momento se han venido aplicando, ¿seguirá siendo correcta esta vía?. Siguiendo a Tedesco (citado por Ottone;2001), podríamos decir que "si bien es cierto la educación es un factor de equidad social, ciertos niveles básicos de equidad social son necesarios para que sea posible educar con posibilidades de éxito".." no se trata solamente de preguntarnos cuál es la contribución de la educación a la equidad social sino, a la inversa. ¿cuánta equidad social es necesaria para que haya una educación exitosa?".

En esta línea argumental podemos pensar entonces que de permanecer este desarrollo inequitativo en educación, se avanzaría en la inequidad con respecto a los ingresos y así mismo ocurre de manera inversa, por tanto el ciclo que se genera no es el círculo de la reproducción de las desigualdades sociales, sino que más allá de esto encontramos el avance de la inequidad en materias salariales y educativas complementándose unas a otras.

Quizás para algunos suene alarmista y exagerado, puesto que si tomamos los niveles medios de crecimiento tanto económico como de acceso educativo han aumentado, por tanto alguien podría señalar que hemos avanzado, a lo cual la respuesta sería: efectivamente Chile ha avanzado en productividad, pero no en equidad.

Creemos importante destacar que nuestro trabajo ha sido centrado en Chile, como una manera de ilustrar las respuestas de la implementación de las políticas económicas en el 
Silva-Peña, I., Chelme-Bustos, A. y Salgado-Labra, I. (2003). Educación y conocimiento:¿Eje de la transformación productiva con equidad? El caso de Chile. RELIEVE:, v. 9, n. 1, p. 73-81.

http://www.uv.es/RELIEVE/v9n1/RELIEVEv9n1 3.htm

ámbito educativo, pero se debe tomas en consideración que estas políticas han sido implementadas a lo largo de toda América Latina. Si bien es cierto Chile ocupa un lugar significativo en el ámbito latinoamericano, corresponderá a quienes tomen este trabajo establecer comparaciones y análisis desde una perspectiva más global, o realizar sus propios análisis en los respectivos países, estableciendo desde ya un canal para el debate y la reflexión.

\section{Bibliografía}

Agacino, Rafael (1994). Cinco Ecuaciones "Virtuosas" del Modelo Económico Chileno y Orientaciones para una Nueva Política Económica. Consultado el 17 de Noviembre de 2001 en http://www.redem.buap.mx/

Baudelot, Christian y Establet, Roger (1998). El nivel educativo sube. Madrid: Editorial Morata

CEPAL (1990). Transformación productiva con Equidad. Santiago, Chile: CEPAL.

CEPAL (1992). Educación y Conocimiento, eje de la transformación productiva con equidad. Santiago, Chile: CEPAL.

CEPAL (1997). La brecha de la equidad. América Latina, El Caribe y la cumbre social. Santiago, Chile: CEPAL.

CEPAL (2000). Equidad, desarrollo y ciudadanía. Santiago, Chile: CEPAL.

Cohen, Ernesto (1997). Educación, eficiencia y equidad. Santiago,Chile: Colección Estudios Sociales CEPAL/OEA Ediciones SUR..

Comité técnico asesor del diálogo nacional sobre la modernización de la educación chilena. (1995). Los desafíos de la educación. Santiago, Chile: Editorial Universitaria.

Martinic, Sergio (2000). Grupos de interés e interacciones comunicativas en las reformas sociales en América Latina. En CEPAL, Hacia dónde va el gasto público en educación? Logros $y$ desafios Volumen II. Serie políticas sociales. Santiago, Chile: CEPAL.

MIDEPLAN (1992). Avanzando en equidad. Un proceso de integración al desarrollo: 19901992. Santiago, Chile: MIDEPLAN.
MIDEPLAN (1998). Situación del Empleo en Chile 1998. Santiago, Chile: MIDEPLAN.

MIDEPLAN (1999). Resultados Encuesta CASEN 1998. Documento $N^{\circ} 5$ Situación de la educación en Chile 1998. Santiago, Chile: MIDEPLAN.

MIDEPLAN (2000). Encuesta CASEN 2000. Consultado el 17 de Noviembre de 2001 en http://www.mideplan.cl

MIDEPLAN (2001a). Indicadores Económicos $y$ Sociales 1990-2000. Consultado el 17 de Noviembre de 2001 en http://www.mideplan.cl/estudios/iesmain.html MIDEPLAN (2001b). Situación de la Educación en Chile 2000. Informe ejecutivo. Santiago, Chile: CEPAL.

MINEDUC (1997). Jornada Completa diurna para todos. Santiago. Chile, MINEDUC.

Ottone, Ernesto (2001). La equidad en América latina en el marco de la globalizacion: la apuesta educativa. En CIDE-PREAL , Economía politica de las reformas educativas en América Latina, pp. 63 a 97. Santiago, Chile: CIDE-PREAL.

UNICEF (2000). Socialización en los primeros años: Repercusiones y alternativas educativas. Ciclo de Debates: Desafios de la Política Educacional $N^{\circ} 5$. Santiago, Chile: UNICEF

\section{Notas}

[1] Dentro de la estructura de ingreso monetario del hogar se contempla el subsidio monetario entendido este como aquellos aportes en efectivo que otorga el Estado a las personas y que abarca a las pensiones asistenciales, subsidio de cesantía, subsidio único familiar, asignaciones familiares, otras transferencias monetarias del estado a los hogares, etc., contempla además el total de los ingresos exceptuando el ingreso por renta de propiedades 
Silva-Peña, I., Chelme-Bustos, A. y Salgado-Labra, I. (2003). Educación y conocimiento:¿Eje de la transformación productiva con equidad? El caso de Chile. RELIEVE:, v. 9, n. 1, p. 73-81.

http://www.uv.es/RELIEVE/v9n1/RELIEVEv9n1 3.htm

\section{$\underline{\text { ABOUT THE AUTHORS / SOBRE LOS AUTORES }}$}

Ilich Silva-Peña (isilva@accioneducativa.cl): Es director del Centro de Investigación y Desarrollo para la Acción Educativa (Chile) y coordina la revista electrónica Praxis (http://www.revistapraxis.cl/, Es Licenciado en educación y actualmente está en su proceso de titulación de Magíster en Ciencias Sociales de la Universidad Arcis. Sus principales áreas de investigación actualmente son "Educación y Género", "Equidad en Educación" e "Historia de la Educación".

Alexis Chelme-Bustos (aachelme@puc.cl): Es Licenciado en Educación por la Universidad Metropolitana de Ciencias de la Educación, candidato a Magíster en Educación Mención Currículo por la misma Universidad, actualmente es doctorando en Ciencias de la Educación por la Universidad Católica de Chile. Becario Conicyt (Comisión Nacional de Ciencia y Tecnología). Sus áreas de interés son: "Teoría Curricular" y "Epistemología de la Educación" y "Equidad en Educación".

Isabel Salgado-Labra (isalgado@accioneducativa.cl): Es directora de perfeccionamiento del Centro de Investigación y Desarrollo para la Acción Educativa (Chile) y coordina la revista electrónica Praxis www.revistapraxis.cl. Es Licenciada en Educación, especialista en Lenguaje y Comunicación, sus áreas de interés son "Objetivos Transversales de la educación", "Educación en Lenguaje y Comunicación "y "Equidad en Educación"

\section{ARTICLE RECORD / FICHA DEL ARTÍCULO}

\begin{tabular}{|c|c|}
\hline $\begin{array}{l}\text { Reference / } \\
\text { Referencia }\end{array}$ & $\begin{array}{l}\text { Silva-Peña, I., Chelme-Bustos, A. y Salgado-Labra, I. (2003). Educación y conocimien- } \\
\text { to:¿Eje de la transformación productiva con equidad?. El caso de Chile. Revista ELec- } \\
\text { trónica de Investigación y EValuación Educativa, v. } 9 \text {, n. } 1 . \\
\text { http://www.uv.es/RELIEVE/v9n1/RELIEVEv9n1 3.htm. Consultado en (poner fecha). }\end{array}$ \\
\hline Title / Título & $\begin{array}{l}\text { Educación y conocimiento: ¿Eje de la transformación productiva con equidad?. El caso } \\
\text { de Chile.[Education and Knowledge: The core of productive transformation with eq- } \\
\text { uity?. The case of Chile] }\end{array}$ \\
\hline Authors / Autores & Silva-Peña, I., Chelme-Bustos, A. y Salgado-Labra, I. \\
\hline $\begin{array}{l}\text { Review / } \\
\text { Revista }\end{array}$ & Revista ELectrónica de Investigación y EValuación Educativa (RELIEVE), v. 9, n. 1 \\
\hline ISSN & $1134-4032$ \\
\hline $\begin{array}{l}\text { Publication date / } \\
\text { Fecha de publicación }\end{array}$ & 2003 (Reception Date: 2 Sept. 2002; Publication Date: 21 Febr. 2003) \\
\hline $\begin{array}{l}\text { Abstract / } \\
\text { Resumen }\end{array}$ & $\begin{array}{l}\text { The objective of this article is to give a review of the results of educational politics in } \\
\text { Chile, of which the core concept has been "productive transformation with equity". } \\
\text { It has established a view on the aspects of productivity and distribution of income in } \\
\text { the different socioeconomic stratums, and it shows the levels of education achieved in } \\
\text { comparison to the incomes earned. } \\
\text { Among the principle conclusions that were found, it is certain that even though produc- } \\
\text { tivity has increased, equity has not, which shows problems with educational develop- } \\
\text { ment in Chile. }\end{array}$ \\
\hline
\end{tabular}


Silva-Peña, I., Chelme-Bustos, A. y Salgado-Labra, I. (2003). Educación y conocimiento:¿Eje de la transformación productiva con equidad? El caso de Chile. RELIEVE:, v. 9, n. 1, p. 73-81.

http://www.uv.es/RELIEVE/v9n1/RELIEVEv9n1_3.htm

\begin{tabular}{|l|l|}
\hline & $\begin{array}{l}\text { El presente artículo tiene por objetivo realizar una revisión de los resultados de políticas } \\
\text { educativas en Chile, que han tenido como eje central el concepto de "transformación } \\
\text { productiva con equidad". } \\
\text { Se establece una mirada desde los aspectos de la productividad y la distribución de in- } \\
\text { gresos en los distintos estratos socioeconómicos, realizando una comparación de los ni- } \\
\text { veles educativos alcanzados de acuerdo al nivel de ingreso. } \\
\text { Dentro de las principales conclusiones y resultados se encuentra que, si bien es cierto, la } \\
\text { productividad ha tenido un aumento, esto no ha sido así en los aspectos de equidad, lo } \\
\text { cual podría señalar algunos inequívocos del desarrollo educativo en Chile. }\end{array}$ \\
\hline $\begin{array}{l}\text { Education, Chile, Educational Policy, Educational Reform, Economy of the educa- } \\
\text { tion, Equity of the Education. } \\
\text { Eesucación, Chile, Política Educacional, Reforma Educativa, Economía de la Educación, } \\
\text { Equidad en Educación }\end{array}$ \\
\hline $\begin{array}{l}\text { Institution / } \\
\text { Institución }\end{array}$ & Centro de Investigación y Desarrollo para la Acción Educativa (Chile) \\
\hline $\begin{array}{l}\text { Publication site / } \\
\text { Dirección }\end{array}$ & http://www.uv.es/RELIEVE \\
\hline Language / Idioma & Español (Title, abstract and keywords in english) \\
\hline
\end{tabular}

\section{Revista ELectrónica de Investigación y $\mathbf{E V}$ aluación Educativa (RELIEVE)}

[ ISSN: 1134-4032 ]

(C) Copyright 2002, RELIEVE. Reproduction and distribution of this articles it is authorized if the content is no modified and their origin is indicated (RELIEVE Journal, volume, number and electronic address of the document). // (C) Copyright 2002, RELIEVE. Se autoriza la reproducción y distribución de este artículo siempre que no se modifique el contenido y se indique su origen (RELIEVE, volumen, número y dirección electrónica del documento). 